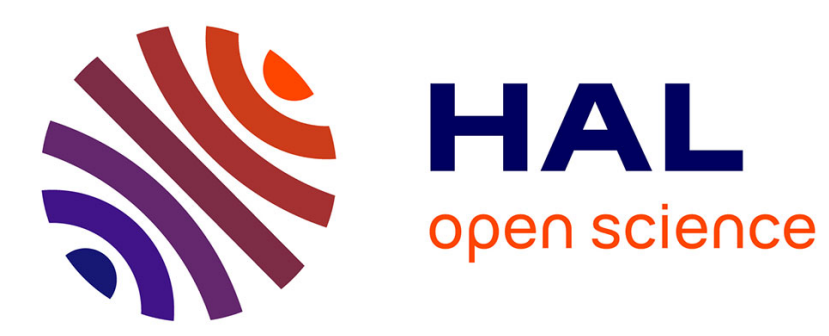

\title{
Technologies numériques 3D et patrimoine, quels enjeux pour demain?
}

Robert Vergnieux

\section{To cite this version:}

Robert Vergnieux. Technologies numériques 3D et patrimoine, quels enjeux pour demain?. Joanne Burgess; Paul-André Linteau. Histoire et Patrimoine, Pistes de recherche et de mise en valeur, UQAM, pp.173-175, 2019, Histoire et Patrimoine. hal-02135952

\section{HAL Id: hal-02135952 \\ https://hal.science/hal-02135952}

Submitted on 21 May 2019

HAL is a multi-disciplinary open access archive for the deposit and dissemination of scientific research documents, whether they are published or not. The documents may come from teaching and research institutions in France or abroad, or from public or private research centers.
L'archive ouverte pluridisciplinaire HAL, est destinée au dépôt et à la diffusion de documents scientifiques de niveau recherche, publiés ou non, émanant des établissements d'enseignement et de recherche français ou étrangers, des laboratoires publics ou privés. 
Technologies numériques 3D et patrimoine, quels enjeux pour demain?

Robert Vergnieux

Commissaire Scientifique de l'exposition ATON-NUM Ancien directeur-fondateur d'Archéovision (UMS 3657-CNRS)

Le domaine des SHS offre de très nombreux champs expérimentaux pour les innovations technologiques. Entre recherche et valorisation, le patrimoine de demain dispose d'atouts exceptionnels que représentent les technologies du numérique. Des appels à projets sur le patrimoine sont régulièrement ouverts, cependant l'obligation qu'ils produisent des innovations technologiques en a freiné le dépôt par les archéologues, historiens, antiquisants, médiéviste, etc. Certes ces experts SHS sont souvent intégrés ponctuellement dans les projets pour valider les contenus patrimoniaux, mais c'est rarement pour participer à la définition des objectifs scientifiques.

1. Le patrimoine en tant que champ expérimental pour l'innovation technologique Nous avons eu la chance de pouvoir concilier sur une trentaine d'années des objectifs scientifiques et des innovations technologiques dans le cadre de projets archéologiques ${ }^{1}$. Les études portaient sur la révolution religieuse menée par Amenhotep IV au milieu du $14^{\mathrm{e}}$ siècle avant notre ère et la mise en place d'une architecture originale dédiée au culte solaire d'Aton. Les programmes successifs ont été développés dans un cadre de transferts et d'innovations technologiques. Chaque étape, conçue comme un maillon indépendant, a obtenu un financement propre. L'objectif

\footnotetext{
${ }^{1}$ Robert Vergnieux, De la CAO ..., p. 173-175
} 
scientifique majeur transcendait tous les projets et consistait à améliorer nos connaissances sur les réformes mises en place sous le règne d'Amenhotep IV ainsi que l'urbanisme qui y était associé. Au gré de l'émergence de nouvelles technologies, nous avons pu proposer, pour chaque programme, des usages originaux débouchant sur des livrables tant égyptologiques que technologiques. Toute la difficulté réside à ne pas succomber au bluff technologique. Trop de projets liés au patrimoine en sont cependant victimes. Évoquons à cet égard le nombre important de thèses issues des milieux informatiques qui traitent de l'assemblage virtuel 3D de tessons de céramique, symbole de l'archéologie ! Les archéologues n'ont rien à faire de tels développements d'autant que la numérisation 3D de millions de tessons ne peut être prise au sérieux tant elle est irréaliste. Pour l'archéologie, les nouvelles technologies ne sont pertinentes que dans la mesure où elles permettent l'acquisition de nouvelles connaissances sur le patrimoine ou bien en favorisent la valorisation.

Nos études ont donc traversé les évolutions technologiques qui à chaque fois ont dynamisé les recherches. Par exemple, mener une étude statistique sur les décors des fragments provenant des murs des temples d'Aton pour obtenir des rapprochements archéologiques entre eux devenait possible avec l'apparition de la micro-informatique. Le CNRS a soutenu alors ces balbutiements pionniers en finançant l'achat de technologie sparow autorisant la manipulation plus de 12000 documents à l'aide d'un micro-ordinateur, ce qui tenait de l'exploit en 1984. Fort de cette base de données opérationnelle nous avons eu accès aux technologies industrielles de l'époque dans le cadre du mécénat technologique et scientifique d'entreprise. C'est ainsi que de super calculateurs et un langage d'intelligence artificielle nous ont été accessibles. Grâce à ces moyens lourds, nous avons pu rapprocher entre eux de nombreux fragments de paroi 
des temples d'Aton et déployer une étude plus générale offrant des résultats sur la compréhension des extensions architecturales situées à l'est du temple d'Amon à Karnak $^{2}$. Ces financements étaient également liés à des opérations de médiation scientifique à destination du grand public. Articles de presse, diffusion dans les médias télévisuels ont été des livrables prévus ayant favorisé les projets suivants.

Une autre convention de mécénat technologique et scientifique encadrait l'étude architecturale des temples de Karnak à l'aide de la CAO qui produisit la première maquette numérique des temples ${ }^{3}$. Quelques années plus tard, nous avons obtenu une copie de ces données par convention nous permettant d'associer le modèle 3D des temples de Karnak et les hypothèses que nous pouvions formuler sur les édifices dédiés à Aton grâce aux bases de données. L'utilisation possible des nouvelles technologies favorisait ainsi l'émergence d'objectifs scientifiques plus ambitieux. Suivre l'évolution architecturale du temple de Karnak dans sa globalité grâce à la précision et la maniabilité de la maquette numérique ; étudier le chantier de construction du IXe pylône de Karnak en s'appuyant simultanément sur plus de 12000 documents devenait des objectifs scientifiques réalistes. Ces développements ont été accompagnés d'expositions, de reportages et d'évènements associés aux journées du patrimoine. Sans reprendre ici tout l'historique des programmes qui se sont succédés sur plus de 30 ans (voir tableau), nous évoquerons certains aspects qui éclairent sur les enjeux méthodologiques et la relation au monde industriel.

\footnotetext{
${ }^{2}$ Marc Albouy, Du Titanic ..., p. 105-132; Robert Vergnieux, Recherches sur les monuments ...

${ }^{3}$ Jean-Claude Golvin, Quelques travaux récents ...
} 
Les technologies liées aux scanners 3D ont connu des développements spectaculaires à partir du moment où les entreprises dans le domaine de l'énergie en ont eu besoin pour la numérisation rétroactive des bâtis industriels existants. Les programmes sur le patrimoine en ont largement bénéficié. Par contre, l'intelligence artificielle en vogue dans les années 1985-1994 fut délaissée par l'industrie ne trouvant pas de champ d'application pertinent. Les usages pionniers effectués par les archéologues restèrent sans lendemains faute d'outil alors disponible ${ }^{4}$. Revenant en force depuis 2015 l'intelligence artificielle retrouve de nouveau un usage méthodologique pertinent pour les SHS, car fortement utilisée dans le monde industriel pour la robotisation et l'interopérabilité du web sémantique. Sans doute, les programmes SHS vont se réapproprier ces axes dans les années à venir.

Mais au-delà des méthodes l'importance stratégique des programmes de recherche en SHS est bien l'accroissement de connaissances sur le patrimoine plutôt que les avancées technologiques qu'ils ont éventuellement occasionnées. À chaque projet pluridisciplinaire, il est fondamental d'expliciter les livrables attendus définissant ainsi la responsabilité scientifique de chacun. En suivant cette stratégie, les interrogations dans un contexte SHS obligent à trouver des réponses méthodologiques innovantes pouvant à leur tour conduire vers des technologiques originales. De pouvoir produire sur les deux versants, SHS et informatique, est une façon de rester en cohérence avec les engagements financiers d'une part et les objectifs scientifiques d'autre part puisque les résultats sont valorisables dans les deux domaines.

\footnotetext{
${ }^{4}$ Sur ces recherches pionnières voir: Jean-Claude Gardin et al., Systèmes experts ...
} 
L'enjeu scientifique majeur reste l'étude de l'urbanisme novateur créé dans la vallée du Nil au XIVe siècle avant notre ère. Cette question n'a pas de relation interdépendante avec les technologies émergentes des années 2000. Cependant, l'évolution des techniques et le passage au numérique ont donc permis d'emprunter des chemins méthodologiques novateurs pour s'interroger sur cette période antique.

L'exposition que nous avons conçue et qui s'est tenue en avril 2016 en Aquitaine s’appuie pour la muséographie sur les outils et fichiers numériques produits pendant ces trente années de recherches ${ }^{5}$. Pour la présentation au public, nous avons décidé d'effectuer une médiation sur la révolution politico-religieuse d'Amenhotep IV et non pas sur les différentes innovations technologiques et méthodes mises en œuvre. Mais nous avons mis en scène cette période de l'histoire en nous servant des différents outils et fichiers numériques 3D utilisés par les programmes scientifiques. Ceci avait pour effet de tester la capacité de nos images scientifiques en tant que support pédagogique.

L'exposition se compose de six projections de films numériques produits à partir des maquettes numériques de travail des équipes scientifiques. Plusieurs dispositifs multimédias permettent aux visiteurs de découvrir des « fac-similés » numériques d'œuvres de cette période et d'explorer l'un des temples restitués. Un module, en holusion, présentait une statuette du couple royal obtenue à partir d'un relevé photogrammétrique effectué lors de l'étude de la mise en couleur de la statuaire égyptienne. Un système en réalité augmentée présente par vidéo projection sur un

\footnotetext{
${ }^{5}$ On peut trouver le guide d'accompagnement à cette adresse : http://atonnum.fr/document/doc/download.php?fic=pdf/mini-guide.pdf
} 
prototype en résine, différentes études de mise en couleur d'un buste d'Akhénaton conservé au Musée du Louvre. Ces deux installations permettaient aux visiteurs de découvrir ces objets du patrimoine mondial ainsi que les technologies les plus évoluées et la façon dont elles peuvent aider la recherche en SHS. Le module de réalité virtuelle est quant à lui issu de plusieurs programmes pluridisciplinaires (Search) menés en partenariat avec l'INRIA. Un module de navigation dans les espaces 3D des temples d'Aton provient des fichiers de travail 3D de l'équipe internationale d'égyptologues qui a travaillé sur le sujet (ANR Aton 3D et programme Aton Numérique). Un navigateur développé dans le cadre d'une autre convention de transfert technologique (EVIA) avec la société Immersion ainsi qu'une table interactive provenant d'un partenariat (Région Aquitaine 2C3D) toujours avec la société Immersion et le CAUE 24, complètent le dispositif.

Cette présentation expérimentale a démontré la faisabilité d'une l'utilisation directe des outils et données 3D des milieux scientifiques à des fins pédagogiques. Sur les vingt journées d'ouverture de l'exposition, ce sont au total 2242 personnes qui ont suivi les visites commentées. D'autres visiteurs, non comptabilisés, ont parcouru l'exposition sans être guidés.

Nous constatons qu'il est tout à fait possible de concilier dans le domaine de la 3D, objectifs patrimoniaux, innovations technologiques et valorisation. Les programmes de recherches construits sur ces trois articulations de financements peuvent s'enchaîner par étapes successives dans la mesure où les livrables sont à chaque fois la base des projets suivants. Enfin il est indispensable qu'une équipe scientifique assure la cohérence entre les différents sources de financements et les objectifs scientifiques à long terme. 
Tableau - Article Vergnieux

\begin{tabular}{|c|c|c|}
\hline Années & Type de projets & Réalisations \\
\hline 1984-1986 & $\begin{array}{l}\text { CAO temples de Karnak } \\
\text { Convention EDF } \\
\text { Programme de Recherche }\end{array}$ & Modèles 3D des temples de Karnak \\
\hline 1985-1992 & $\begin{array}{l}\text { Intelligence Artificielle / Talatat } \\
\text { Convention EDF } \\
\text { Programme de Recherche } \\
\end{array}$ & $\begin{array}{c}\text { Bases de règles et base de faits } \\
\text { Base de données sur les pierres des temples } \\
\text { d'Aton }\end{array}$ \\
\hline 1987-1991 & $\begin{array}{l}\text { « Les bâtisseurs de Karnak » } \\
\text { Exposition Itinérante } \\
\end{array}$ & Poursuite modélisation des temples de Karnak \\
\hline 1993-1994 & $\begin{array}{l}\text { CAO en Archéologie } \\
\text { Convention EDF } \\
\text { Programme de Recherche }\end{array}$ & $\begin{array}{c}\text { Transfert technologique des outils de CAO dans } \\
\text { une équipe d'archéologues (Bordeaux) } \\
\text { Transfert des bases de données 3D - Karnak }\end{array}$ \\
\hline 1998 & $\begin{array}{l}\text { «Égyptologie, le rêve et la } \\
\text { science » } \\
\text { Exposition - Electra - Paris }\end{array}$ & $\begin{array}{l}\text { Dessins des murs des temples d'Aton de } \\
\text { Karnak - IXe pylône de Karnak }\end{array}$ \\
\hline $2000-2005$ & $\begin{array}{c}\text { ICONIC } \\
\text { Programme de Recherche }\end{array}$ & $\begin{array}{c}\text { Restitution du Sanctuaire d'Aton et étude du } 9^{\mathrm{e}} \\
\text { Pylône de Karnak }\end{array}$ \\
\hline 2000 & $\begin{array}{l}\text { « Du Nil à Rome » } \\
\text { Exposition - Itinérante }\end{array}$ & Modèle 3D du chantier de l'obélisque unique \\
\hline $2007-2009$ & $\begin{array}{c}\text { SEARCH } \\
\text { Agence Nationale de la Recherche }\end{array}$ & $\begin{array}{l}\text { Développement d' outils de photogrammétrie et } \\
\text { de réalité augmentée }\end{array}$ \\
\hline 2008 & $\begin{array}{l}\text { «Akhenaton et Nefertiti » } \\
\text { Exposition - Musée d'Art et } \\
\text { d'Histoire de Genève }\end{array}$ & Modèle 3D de la ville d'Amarna \\
\hline $2008-2010$ & $\begin{array}{c}\text { ATON 3D (ANR) } \\
\text { Programme de Recherche }\end{array}$ & $\begin{array}{l}\text { Modèle 3D du Gm-Pa-Aton + Buste } \\
\text { d'Amenhotep IV-Akhenaton }\end{array}$ \\
\hline 2011 & $\begin{array}{l}\text { «Akhénaton. La Construction } \\
\text { de la Lumière » } \\
\text { Exposition - Espace Vaucluse } \\
\text { Avignon }\end{array}$ & $\begin{array}{l}\text { Modélisation volumique des colosses du Gem- } \\
\qquad \text { pa-Aton }\end{array}$ \\
\hline $2010-2013$ & $\begin{array}{c}2 \mathrm{C} 3 \mathrm{D} \\
\text { Transfert technologique } \\
\end{array}$ & $\begin{array}{c}\text { Intégration de l'usage d'une table collaborative } \\
\text { associée aux modèles 3D }\end{array}$ \\
\hline 2011-2016 & $\begin{array}{c}\text { ATON-Numérique } \\
\text { Programme de recherche - Région }\end{array}$ & $\begin{array}{l}\text { Modèle 3D : Est de Karnak, ville d'Amarna } \\
\text { (jardins , Statuaire des temples. }\end{array}$ \\
\hline 2012 & $\begin{array}{c}\text { «In the light of Amarna » } \\
\text { Exposition - Musée de Berlin }\end{array}$ & $\begin{array}{l}\text { Prototypage du buste d'Amenhotep IV - } \\
\text { Akhenaton (Louvre) }\end{array}$ \\
\hline 2013-2015 & $\begin{array}{c}\text { EVIA } \\
\text { Transfert technologique - Région }\end{array}$ & Interface de visualisation des modèles 3D \\
\hline 2016 & $\begin{array}{c}\text { «ATON-NUM } » \\
\text { Exposition - Bordeaux } \\
\end{array}$ & $\begin{array}{l}\text { Films HD, holusion, site web ; crowd-sourcing ; } \\
\text { Revealing Flashlight }\end{array}$ \\
\hline 2017 & $\begin{array}{l}\text { «ATON-NUM » } \\
\text { Exposition - Lille }\end{array}$ & $\begin{array}{l}\text { Automatisation des applis multimédias pour une } \\
\text { meilleure diffusion. }\end{array}$ \\
\hline
\end{tabular}

Tableau :

Titre Légende : Tableau non exhaustif qui montre la succession d'événements qui ont contribué aux financements de la recherche sur le règne d'Amenhotep IV-Akhenaton. Dans la première colonne sont indiquées les années concernées. Dans la seconde colonne, la nature de l'événement. Et enfin dans la troisième colonne les principales réalisations auxquels les financements ont contribué. 
Encadré : - Liste des structures ayant contribuées aux financements des différents projets

CNRS : Département puis Institut SHS du Centre National de la Recherche Scientifique

MTS EDF : Mécénat Technologique et Scientifique d'EDF

IMMERSION :Société Immersion - Bordeaux

CASDEN : Mécénat Banque Populaire

CRA : Conseil Régional d'Aquitaine (puis Nouvelle Aquitaine)

ANR : Agence Nationale pour la Recherche

UBM : Université Bordeaux Montaigne

Musée d'Art et d'Histoire de Genève

Association Égyptologique de Gironde

Ägyptisches Museum und Papyrussammlung (Staatliche Museum zu Berlin)

- Liste des principaux partenaires ayant contribué aux recherches et aux développements technologiques :

ARCHEOTRANSFERT (ADERA)

LABRI - Université de Bordeaux - CNRS

Département TIEM (EDF CLAMART)

INRIA

HUMA-NUM

Musée d'Art et d'Histoire de Genève

Musée du Louvre - Département des Antiquités Égyptiennes

Université de Cambridge (1E_E_S__A_m_a_r_n_a__E_x_p_e_d_i_t_i_o_n__)

Université Paul Valéry Montpellier 3

Université de Liège - FNRS

Centre d'Egyptologie - Avignon (puis Montségur)

Centre Franco-Egyptien d'Etudes des Temples de Karnak 


\section{Bibliographie}

Albouy, Marc, Du Titanic à Karnak: l'aventure du mécénat technologique, (Paris, France: Dunod, 1994)

Albouy, Marc, Henri Boccon-Gibod, Jean-Claude Golvin, Jean-Claude Goyon, et Philippe Martinez, Karnak. Le temple d'Amon restitué par l'ordinateur (Solar, 1989)

Gardin, Jean-Claude, Olivier Guillaume, et P.-Q. Herman, Systèmes experts et sciences humaines: le cas de l'archéologie, (Paris, France: Eyrolles, 1987)

Golvin, Jean-Claude, 'Quelques travaux récents du Centre Franco-Egyptien de Karnak', CRAIBL : Comptes Rendus de l'Académie Des Inscriptions et Belles-Lettres, 1988, 576-599

Vergnieux, Robert, 'De la CAO à la photogrammétrie: 30 ans d'exploration des nouveaux usages de la 3D pour les SHS', dans les Actes Du Colloque Virtual Retrospect 2013, Robert Vergnieux et Caroline Delevoie, (ed. Scient.), Archéovision 6 (Pessac, France: Ausonius Editions, 2015)

Vergnieux, Robert, 'L'usage de la 3D en Archéologie', dans Robert Vergnieux et Nigel Strudwick (ed . Scient.) Information technology and Egyptology in 2008: acte du Computer Working Group of the International Association of Egyptologists (Informatique et Egyptologie), Vienna, 8-11 July, 2008 (Piscataway, N.J., Etats-Unis d'Amérique: Gorgias Press, 2008), pp. 147-154

Vergnieux, Robert, Recherches sur les monuments Thébains d'Amenhotep IV à l'aide d'outils informatiques : Méthodes et résultats, Cahiers de la Société d'égyptologie; v. 4, Fasc. 1-2 (Genève: Société d'égyptologie, 1999) 


\section{Illustrations}

Figure 01 : Entrée de l'exposition. Deux piliers de 1'Hôtel de Région sont décorés avec l'impression sur toile d'un colosse d'Amenhotep IV obtenue à partir du modèle 3D développé par l'équipe scientifique (Archeotransfert-Archeovision).

Figure 02 : Installation du dispositif multimédia de projection vidéo sur une réplique du buste d'Amenhotep IV. L'installation utilise les logiciels développés par le programme de recherche ainsi que la réplique du buste produite dans le cadre du programme de recherche Aton-numérique (INRIA- Archéovision).

Figure 03 : Image issue du modèle 3D de la partie centrale de la ville d'Amarna. Ce modèle 3D développé sur de nombreuses années par la recherche est utilisé pour produire les visuels de l'exposition (Archéotransfert).

Figure 04 : Tests d'utilisation dans le contexte de la recherche d'une table collaborative. Une variante de cette table sera utilisée par le publique lors de l'exposition (Immersion).

Figure 05 : Visuels des différentes manifestations de médiation des projets de recherches. 


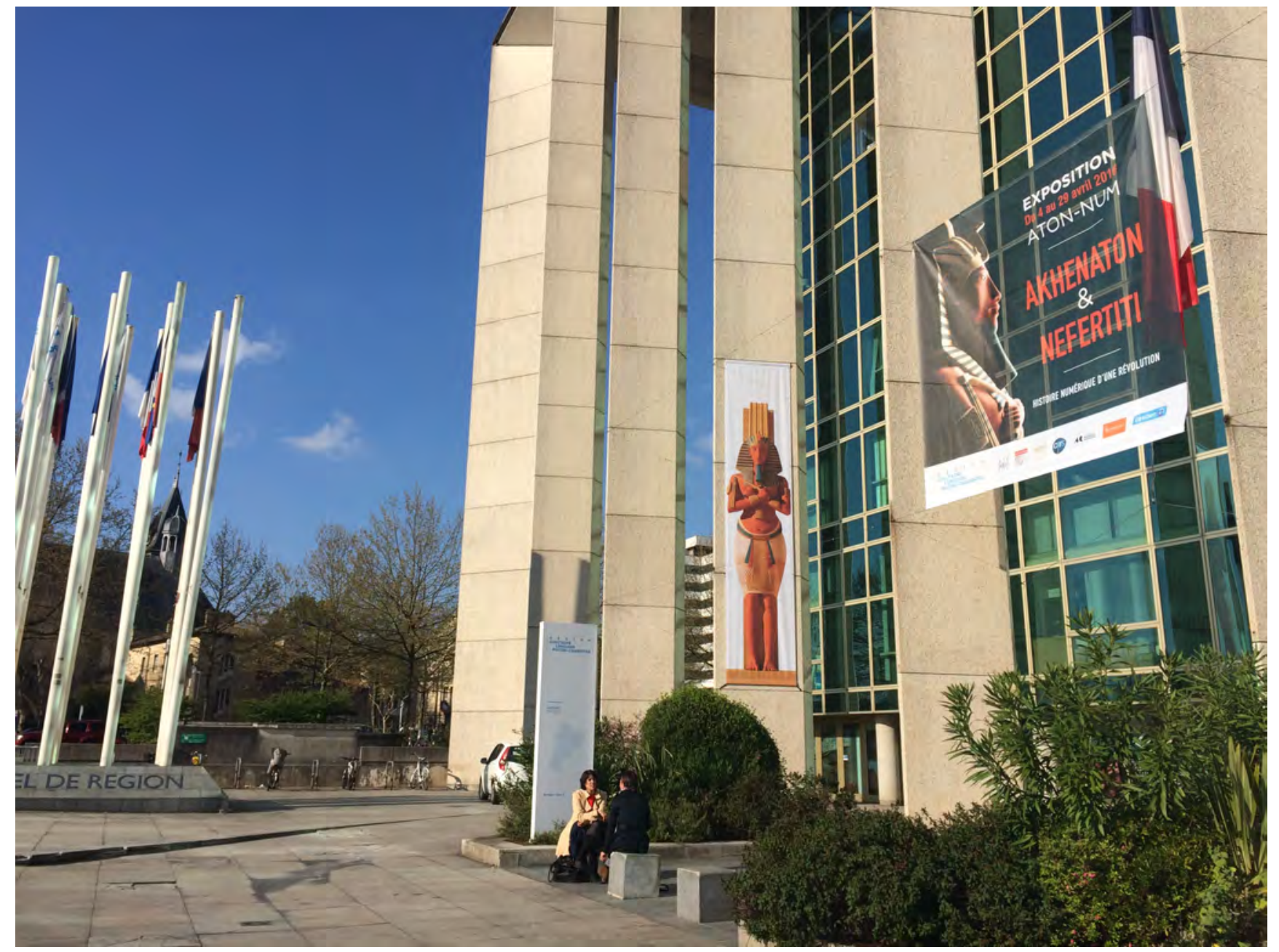

Figure 01

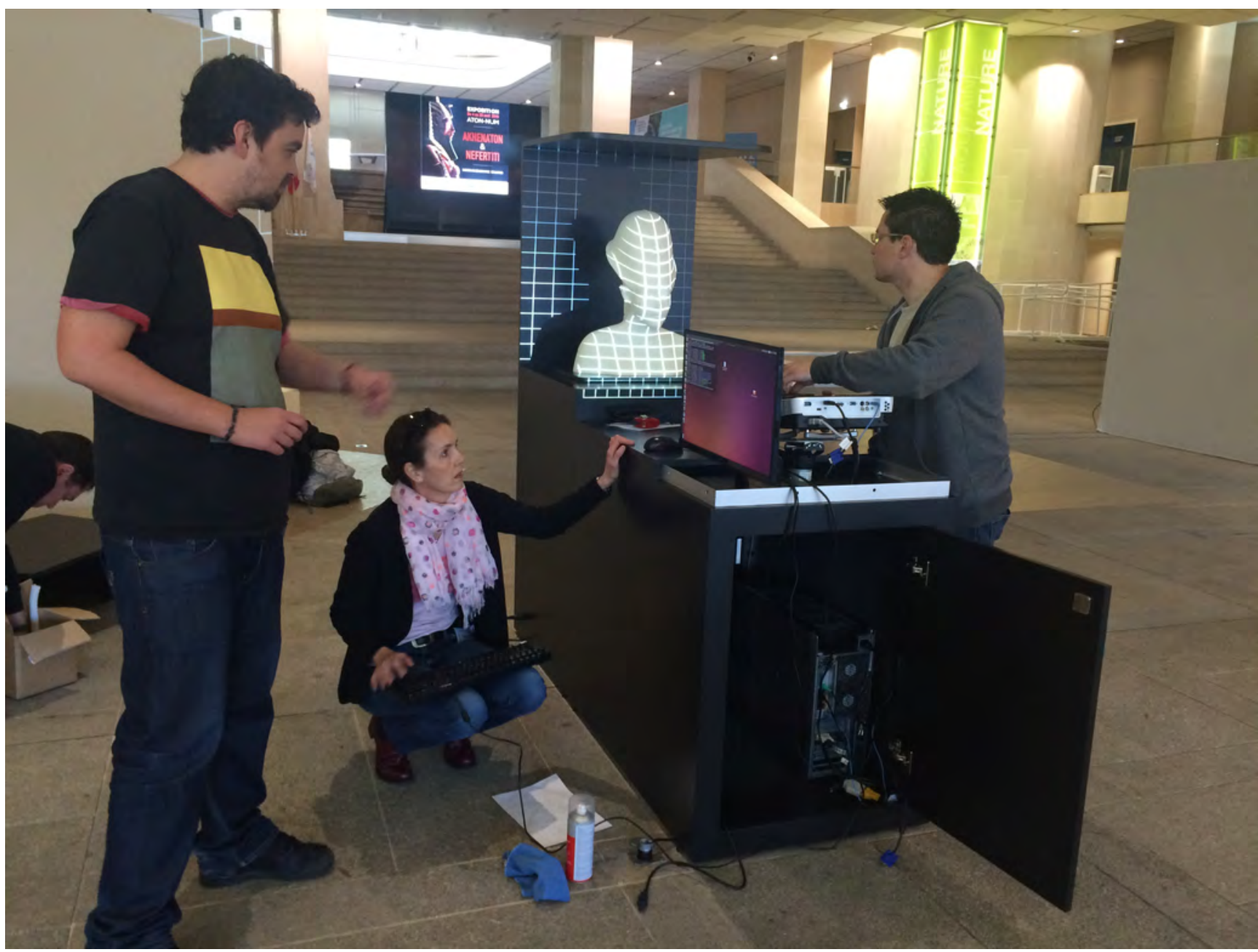




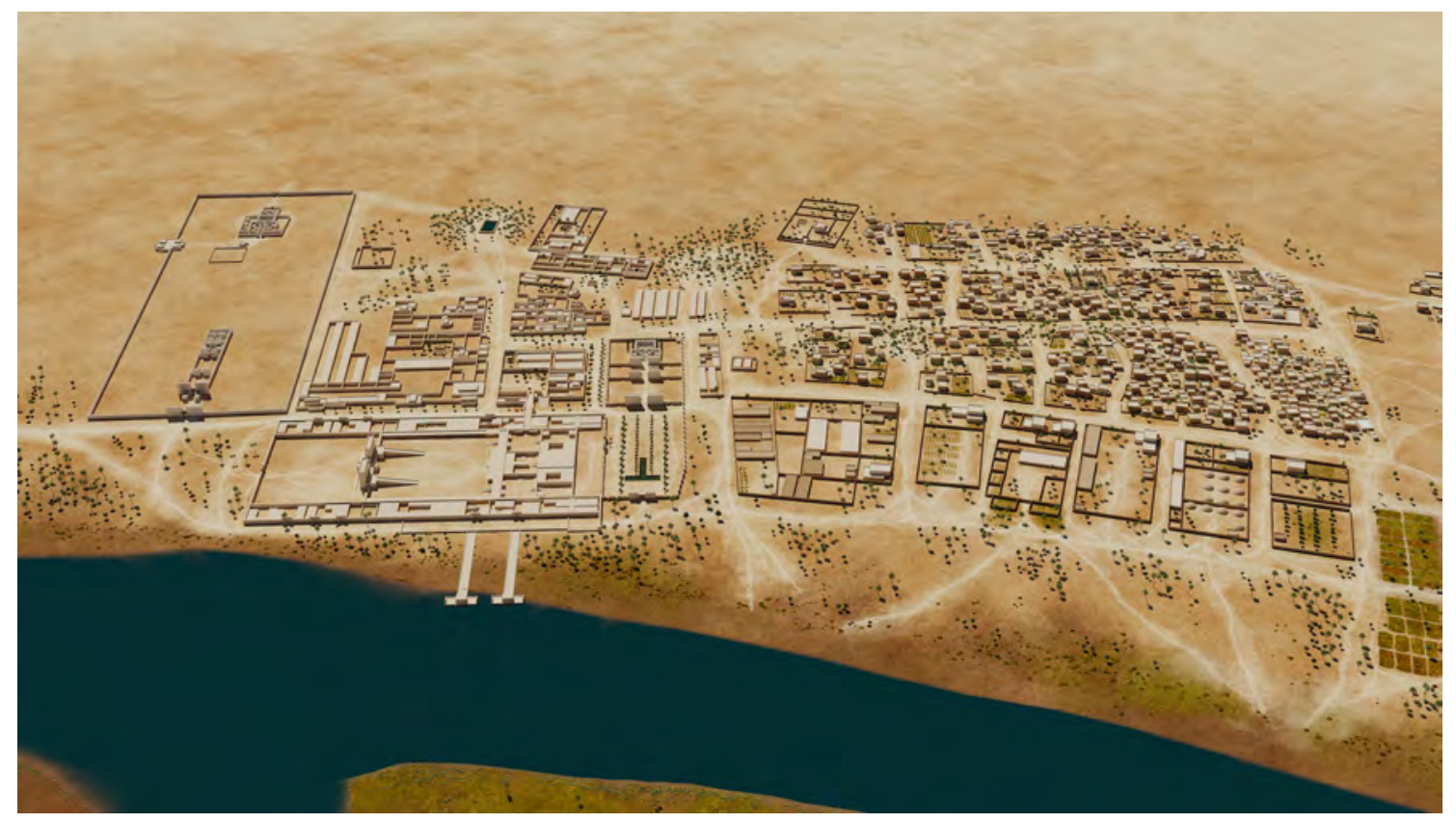

Figure 03

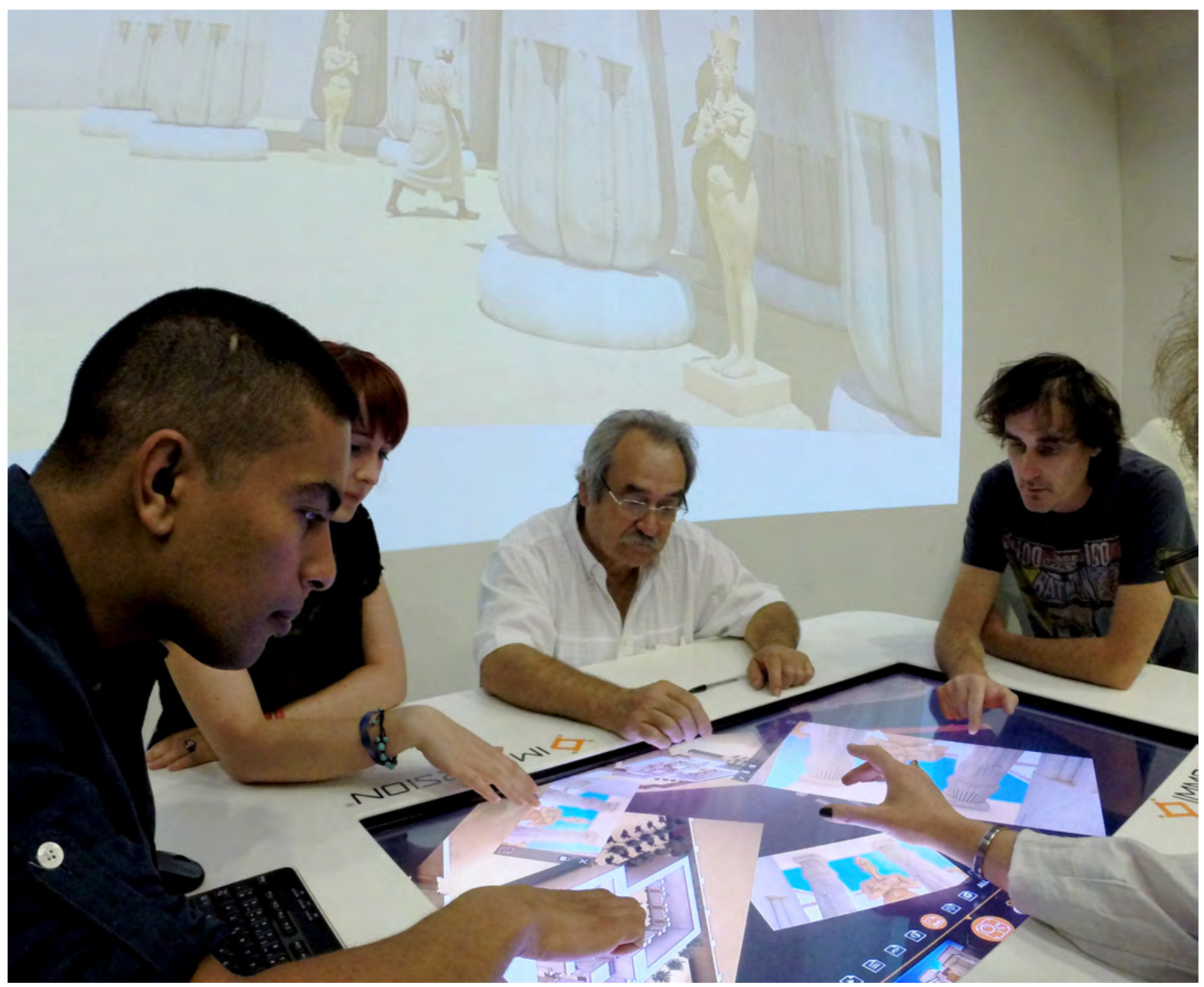

Figure 04 


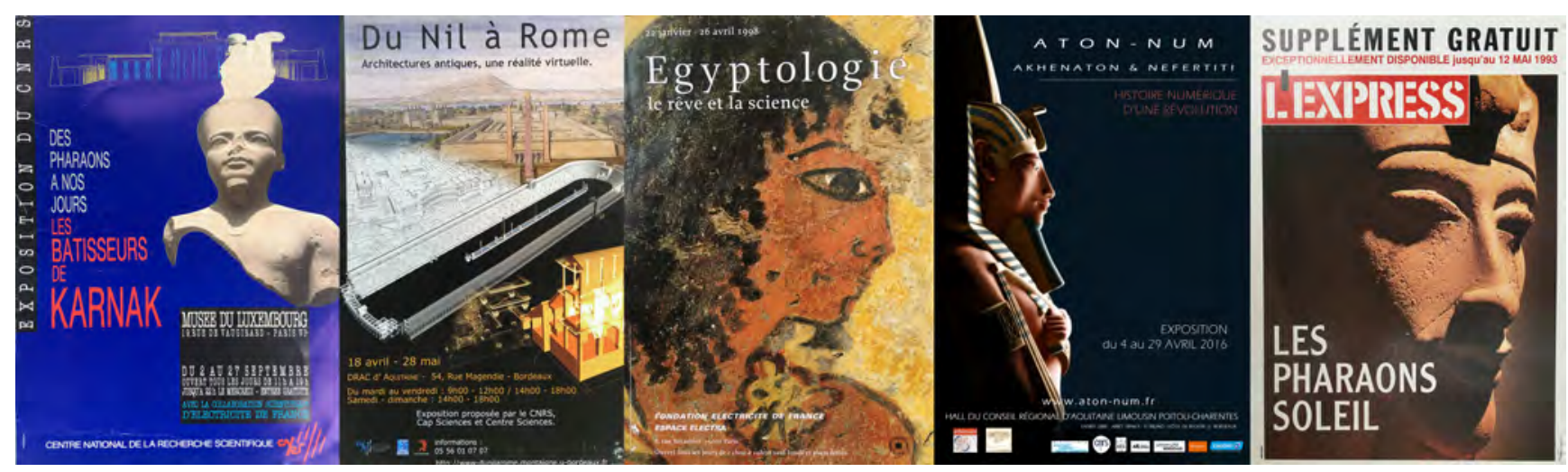

Figure 05 